\title{
Comparison of EUS-guided conventional smear and liquid-based cytology in pancreatic lesions: A systematic review and meta- analysis
}

\section{다 (i) $(-)$}

\section{Authors}

Saurabh Chandan*, ${ }^{*}$, Babu P. Mohan*,2, Shahab R. Khan ${ }^{3}$, Andrew Ofosu4 ${ }^{4}$, Amaninder S. Dhaliwal ${ }^{5}$, Aun R. Shah ${ }^{6}$, Neil Bhogal $^{6}$, Harmeet S. Mashiana ${ }^{6}$, Simran S. Mashiana7, Lena L. Kassab ${ }^{8}$, Suresh Ponnada ${ }^{9}$, Antonio Facciorusso ${ }^{10}$, Ishfaq Bhat ${ }^{6}$, Shailender Singh ${ }^{6}$, Benjamin L. Witt ${ }^{11}$, Douglas G. Adler ${ }^{2}$

Institutions

1 Division of Gastroenterology and Hepatology, $\mathrm{CHI}$ Creighton University Medical Center, Omaha, Nebraska, USA

2 Division of Gastroenterology and Hepatology, University of Utah School of Medicine, Salt Lake City, Utah, USA

3 Section of Gastroenterology, Rush University Medical Center, Chicago, Illinois, USA

4 Division of Gastroenterology \& Hepatology, Stanford University, Stanford, California, United States

5 Division of Digestive Diseases \& Nutrition, University of South Florida, Tampa, Florida, USA

6 Gastroenterology and Hepatology, University of Nebraska Medical Center, Omaha, Nebraska, USA

7 Pathology \& Microbiology, University of Nebraska Medical Center, Omaha, Nebraska, USA

8 Internal Medicine, Mayo Clinic, Rochester, Minnesota, USA

9 Internal Medicine, Carilion Roanoke Memorial Hospital, Roanoke, Virginia, USA

10 Gastroenterology Unit, Department of Medical Sciences, University of Foggia, Foggia, Italy

11 Cytopathology Section, University of Utah School of Medicine, Salt Lake City, Utah, USA

submitted 6.5.2020

accepted after revision 7.7.2020

Bibliography

Endoscopy International Open 2020; 08: E1611-E1622

DOI 10.1055/a-1240-0027

ISSN 2364-3722

(c) 2020. The Author(s).

This is an open access article published by Thieme under the terms of the Creative Commons Attribution-NonDerivative-NonCommercial License, permitting copying and reproduction so long as the original work is given appropriate credit. Contents may not be used for commecial purposes, or adapted, remixed, transformed or built upon. (https://creativecommons.org/licenses/by-nc-nd/4.0/)
Corresponding author

Douglas G. Adler MD, FACG, AGAF, FASGE, Professor of Medicine, Director of Therapeutic Endoscopy, Director, GI fellowship Program, Gastroenterology and Hepatology, University of Utah School of Medicine, Huntsman Cancer Center, 30N 1900E 4R118, Salt Lake City, Utah 84132, United States

Fax: +1-801-581-8007

Douglas.adler@hsc.utah.edu

丹 Supplementary material is available under https://doi.org/10.1055/a-1240-0027

\section{ABSTRACT}

Background and study aims Endoscopic ultrasound (EUS)-guided fine-needle aspiration (EUS-FNA) has limitations of inadequate sampling and false-negative results for malignancy. It has been performed using conventional smear (CS) cytology with rapid on-site evaluation (ROSE) with reasonable diagnostic accuracy. An alternative to ROSE is liquid-based cytology (LBC). Commonly used LBC techniques include precipitation-based (SurePath ${ }^{\mathrm{TM}}$ ) and filtration-based (ThinPrep ${ }^{\circledR}$, CellPrep ${ }^{\circledR}$ ). Data regarding the diagnostic efficacy of LBC compared with CS are limited. Methods Multiple databases were searched through March 2020 to identify studies reporting diagnostic yield of EUS-guided CS and LBC in pancreatic lesions. Pooled diagnostic odds and rates of performance for the cytologic diagnoses of benign, suspicious, and malignant lesions were calculated. Diagnostic efficacy was evaluated by pooled rates of accuracy, sensitivity, specificity, positive predictive value (PPV) and negative predictive value (NPV). Results Nine studies with a total of 1308 patients were included in our final analysis. Pooled diagnostic odds of CS cytology were 1.69 (Cl 1.02-2.79) and 0.39 (Cl 0.19-0.8) for malignant lesions when compared to filtration-based and precipitation-based LBC techniques, respectively. For CS, precipitation-based and filtration-based LBC, pooled diag-

\footnotetext{
* These authors contributed equally.
} 
nostic accuracy was $79.7 \%, 85.2 \%, 77.3 \%$, sensitivity was $79.2 \%, 83.6 \%$, 68.3\%, and specificity was $99.4 \%$, 99.5\%, $99.5 \%$, respectively.

Conclusions The precipitation-based LBC technique (SurePath $^{\mathrm{TM}}$ ) had superior diagnostic odds for malignant pancreatic lesions compared with CS cytology in the absence of ROSE. It showed superior accuracy and sensitivity, but comparable specificity and PPV. Diagnostic odds of CS cytology in the absence of ROSE were superior to the filtration-based LBC technique (ThinPrep ${ }^{\circledR}$, Cellprep ${ }^{\circledR}$ ) for diagnosing malignant pancreatic lesions.

\section{Introduction}

Endoscopic ultrasound (EUS)-guided fine-needle aspiration (FNA) was first described in 1992 and remains a safe, well-established, and first-line diagnostic tool for the evaluation of solid pancreatic lesions [1]. With regard to diagnosing solid pancreatic lesions, its sensitivity and specificity has been reported to be $86.8 \%$ and $95.8 \%$, respectively [2]. The most common solid pancreatic lesions are pancreatic adenocarcinoma (PAC) followed by neuroendocrine tumor (NET) and solid pseudopapillary tumor (SPT) [3]. PAC is the seventh leading cause of global cancer deaths in industrialized countries and the third most common in the United States. PAC remains one of the most lethal malignancies, with a dismal prognosis and mortality/incidence ratio of $94 \%[4,5]$. Therefore, establishing early and accurate diagnosis remains paramount.

EUS-guided pancreatic sampling can be performed by obtaining fine-needle aspiration (EUS-FNA) as well as core fineneedle biopsy samples (EUS-FNB). Some of the limitations of EUS-FNA include risks of inadequate sampling and false-negative results for malignancy in up to $20 \%$ to $40 \%$ of cases [6, 7]. EUS-FNB needles, on the other hand, allow sampling of a larger amount of tissue and better preservation of cell architecture than an aspirate. Several studies have shown comparable diagnostic accuracy of EUS-FNA and EUS-FNB in solid pancreatic lesions, with the exception of fewer needle passes needed to obtain an adequate sample using the EUS-FNB technique [8-11]. Recently Sweeney et al also showed comparable diagnostic yield of EUS-FNA, FNB, and combined FNA and FNB in solid pancreatic lesions [12].

To overcome the limitations of EUS-FNA, ROSE has gained popularity across health centers, especially in the United States. This technique involves evaluation of direct smears obtained at the point of care in the endoscopy suite by an in-house cytopathologist [13]. Studies have suggested a $3.5 \%$ increase in adequacy rates [14] and $8 \%$ increase in sensitivity [15] for EUSFNA of solid pancreatic lesions with the addition of this technique. Other factors that influence the diagnostic yield of EUSguided sampling include operator experience or expertise, tumor location as well as size and characteristics of the lesion [16].

An alternative to ROSE is LBC in which FNA samples are collected in a liquid medium. This technique makes samples less vulnerable to contamination or artifacts and allows for optimal cell preservation as there is immediate fixation and storage of collected samples at room temperature. In addition, no special technical training or skills are required for collecting the mate- rial in the fixative solution, which can solve the problem of sample assessment in the absence of ROSE [17]. The LBC technique is further divided into filtration-based (ThinPrep ${ }^{\circledR}$, CellPrep ${ }^{\circledR}$ ) and precipitation-based (SurePath ${ }^{\mathrm{TM}}$ ) [18].

Studies have reported higher accuracy of LBC over CS cytology in diagnosis of cervical, gallbladder, and biliary cancers; however, data regarding its applicability in pancreatic lesions are limited $[19,20]$. We evaluated the diagnostic accuracy of filtration-based and precipitation-based LBC compared to CS cytology in pancreatic lesions using meta-analysis.

\section{Methods}

\section{Search strategy}

The literature was searched by a medical librarian for publications regarding the concepts of liquid-based or smear-based cytology for EUS-guided fine-needle aspiration of pancreatic lesions. The search strategies were created using a combination of keywords including "SurePath”, “ThinPrep”, "conventional Papanicolaou smear", "endoscopic ultrasonography-guided fine-needle aspiration", "liquid-based cytology", "pancreatic lesion" and standardized index terms. Searches were performed during March 2020 in ClinicalTrials.gov, Ovid EBM Reviews, Ovid Embase (1974+), Ovid Medline (1946+including epub ahead of print, in-process \& other non-indexed citations), Scopus $(1970+)$ and Web of Science $(1975+)$. Results were limited to English language. The full search strategy is available in Supplementary Appendix A. The MOOSE checklist was followed and is provided as Supplementary Appendix B [21, 22]. Reference lists of evaluated studies were examined to identify other studies of interest.

\section{Study selection}

We included studies that evaluated the diagnostic efficacy of conventional smear-based cytology with LBC in EUS-guided FNA sampling of pancreatic lesions. Studies were included irrespective of inpatient/ outpatient setting, study sample size, follow-up time, and geography as long as they provided the appropriate data needed for the analysis.

Our exclusion criteria were studies performed in the pediatric population (age <18 years) and studies not published in English language. In cases of multiple publications from a single research group reporting on the same patient, same cohort and/or overlapping cohorts, data from the most recent and/or most appropriate comprehensive report were retained. Authors did not need to be contacted for clarification of possible study-cohort overlap, as all included studies were published as 
full manuscripts. The retained studies were decided by two authors (BPM, SC) based on the publication timing (most recent) and/or the sample size of the study (largest). In situations where a consensus could not be reached, overlapping studies $[23,24]$ were included in the final analysis and any potential effects were assessed by sensitivity analysis of the pooled outcomes by leaving out one study at a time. Studies reporting outcomes of LBC were also reviewed separately by another author (SM) with expertise in pathological sciences prior to inclusion in our final analysis,

\section{Data abstraction and quality assessment}

Data on study-related outcomes from the individual studies were abstracted independently onto a standardized form by at least three authors (BPM, SRK, AS). Authors SC, ARS, ASD, NB cross-verified the collected data for possible errors and two authors (SRK, SC) did the quality scoring independently. The Newcastle-Ottawa scale for cohort studies was used to assess the quality of studies [25]. This quality score consisted of eight questions, the details of which are provided in Supplementary Table 1.

\section{Outcomes assessed}

Two outcomes were assessed: (1) pooled odds of CS cytology compared with LBC techniques in diagnosing benign, suspicious and malignant pancreatic lesions with analysis of outcomes of CS cytology with both precipitation-based and filtration-based methods of LBC; and (2) pooled diagnostic performance in terms of accuracy, sensitivity, specificity, PPV and NPV of CS and LB cytology.

\section{Statistical analysis}

We used meta-analysis techniques to calculate the pooled estimates in each case following the methods suggested by DerSimonian and Laird using the random-effects model [26]. When the incidence of an outcome was zero in a study, a continuity correction of 0.5 was added to the number of incident cases before statistical analysis. [27]. We assessed heterogeneity between study-specific estimates by using Cochran Q statistical test for heterogeneity, 95\% confidence interval $(\mathrm{Cl})$, which deals with the dispersion of the effects, and the $\mathrm{I}^{2}$ statistics [27-29]. In this, values $<30 \%, 30 \%$ to $60 \%, 61 \%$ to $75 \%$, and $>75 \%$ were suggestive of low, moderate, substantial, and considerable heterogeneity, respectively. We reported our primary outcomes as pooled odds ratio (OR) between CS cytology and LBC techniques where OR denotes the proportional probability of diagnosing a malignant and/or benign and/or a suspicious lesion with one technique as compared to the other.

Publication bias was ascertained, qualitatively, by visual inspection of funnel plot and quantitatively, by the Egger test [28]. When publication bias was present, further statistics using the fail-Safe $\mathrm{N}$ test and Duval and Tweedie's 'Trim and Fill' test was used to ascertain the impact of the bias [30]. Three levels of impact were reported based on the concordance between the reported results and the actual estimate if there were no bias. The impact was reported as minimal if both versions were estimated to be same, modest if effect size changed substantially but the final finding would still remain the same, and severe if basic final conclusion of the analysis is threatened by the bias [31]. $P<0.05$ was used "a-priori" to define significance between the groups compared.

All analyses were performed using Comprehensive Meta-Analysis (CMA) software, version 3 (BioStat, Englewood, New Jersey, United States).

\section{Results}

\section{Search results and population characteristics}

From an initial pool of 756 studies, 454 records were screened and 48 full-length articles were assessed. A total of nine studies (including 1308 patients) were included in the final analysis [18,32-39]. None of the included studies used ROSE. Four studies originated in Korea, two in China, and one each from the Netherlands, the United States, and Japan. Three studies used the precipitation-based technique of LBC [32-34] whereas six used the filtration-based technique (five studies used ThinPrep ${ }^{\circledR}$ and one used CellPrep ${ }^{\circledR}$ [18]). The schematic diagram demonstrating our study selection is shown in Supplementary Fig. 1.

Baseline population characteristics were comparable between the CS and LBC cohorts. The mean and/or median age ranged from 54.9 to 74 years. The majority of the final diagnosis were reported as pancreatic ductal adenocarcinoma (PDAC) in 544 cases. Further details along with the population characteristics are listed in $\nabla$ Table $\mathbf{1} \mathbf{a}, \mathbf{b}$.

\section{Characteristics and quality of included studies}

Six studies were prospective in design, [18, 23, 32, 35, 39, 40] and the remaining were retrospective. The detailed study quality evaluation is presented in Supplementary Table 1. Based on the New-Castle Ottawa scoring system, seven studies were considered to be of high quality and two studies were considered to be of medium quality. There were no low-quality studies.

\section{Definition of cytological diagnosis}

Cytologic diagnoses in the techniques were made according to the Papanicolaou Society of Cytopathology (PSC) guidelines and classified into three categories: (1) benign, defined as the presence of sufficient cellularity for making diagnoses of benign tumors; (2) suspicious for malignancy, meaning there were some typical features of a specific malignant neoplasm, but quantity was insufficient; and (3) malignant, a group of tumors that clearly show malignant cytologic features.

\section{Meta-analysis outcomes}

1. Pooled odds of CS cytology compared with LBC in diagnosing benign, suspicious and malignant lesions:

a) CS cytology performed comparably to LBC in diagnosing benign and suspicious pancreatic lesions $[O R=1.1(0.7-$ 1.75); $P=0.7,1.23(0.96-1.59) ; P=0.1]$ when combining the data from both the filtration and precipitation-based methods for LBC. 


\begin{tabular}{|c|c|c|c|c|c|c|c|c|c|c|c|}
\hline $\begin{array}{l}\overline{\frac{E}{0}} \\
\text { ज̃ }\end{array}$ & $\stackrel{9}{9}$ & 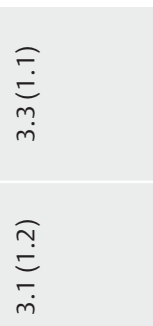 & 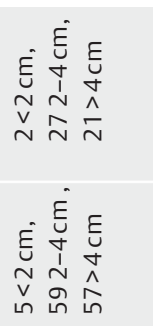 & 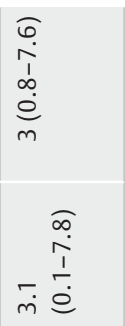 & 1 & \multicolumn{2}{|l|}{ 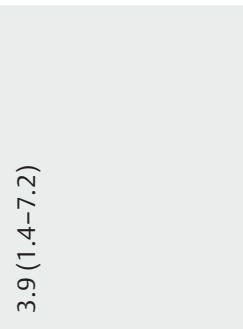 } & $\begin{array}{l}\infty \\
0 \\
x \\
0 \\
0 \\
0 \\
1 \\
0 \\
0 \\
x \\
0 \\
0 \\
0\end{array}$ & 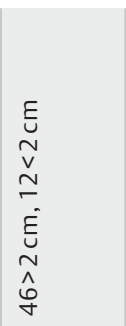 & $\frac{\hat{m}}{\frac{m}{+1}}$ & $\begin{array}{l}\stackrel{N}{N} \\
+1 \\
\stackrel{+}{m}\end{array}$ \\
\hline \multirow{2}{*}{ 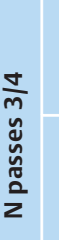 } & $\stackrel{\Xi}{ }$ & $\frac{1}{8}$ & & $\stackrel{\widehat{o}}{\stackrel{i}{1}}$ & 1 & 1 & 1 & 1 & & & 1 \\
\hline & 气 & $\stackrel{\aleph}{\curvearrowright}$ & $\underset{\substack{m \\
\stackrel{d}{d}}}{m}$ & $\stackrel{\overrightarrow{0}}{\dot{p}} \stackrel{\stackrel{1}{=}}{=}$ & 1 & 1 & 1 & 1 & $\begin{array}{l}\infty \\
1 \\
\sim \\
\infty \\
m \\
m\end{array}$ & $\begin{array}{l}\frac{\tilde{n}}{\mathfrak{n}} \\
\underset{m}{n}\end{array}$ & 1 \\
\hline 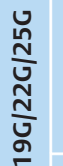 & $\stackrel{9}{9}$ & $\frac{8}{\sigma}$ & $\begin{array}{l}\frac{1}{2} \\
\frac{1}{0}\end{array}$ & & 1 & & & & $\frac{\stackrel{d}{\frac{1}{f}}}{\frac{0}{\frac{0}{0}}}$ & & \\
\hline$\frac{\varrho}{\bar{z}}$ & 气 & 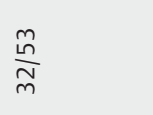 & $\frac{\stackrel{n}{\frac{n}{n}}}{\frac{m}{0}}$ & 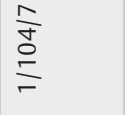 & 1 & $\begin{array}{l}0 \\
\stackrel{N}{N}\end{array}$ & $\underset{N}{\tilde{N}}$ & $\underset{N}{\mathbb{N}}$ & $\frac{\stackrel{d}{\frac{d}{d}}}{\frac{m}{d}}$ & $\underset{\stackrel{n}{\stackrel{N}{N}}}{\stackrel{N}{N}}$ & $\begin{array}{l}\text { J্ } \\
\underset{N}{N}\end{array}$ \\
\hline \multirow[b]{2}{*}{$\frac{\omega}{\Sigma}$} & $\stackrel{M}{9}$ & $\frac{\hat{m}}{\stackrel{m}{\infty}}$ & & $\underset{\infty}{\stackrel{\sigma}{+}}$ & & & & & & 1 & \\
\hline & $\breve{u}$ & 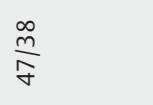 & 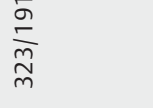 & $\frac{g}{\hat{r}}$ & $\frac{\sqrt{2}}{\sqrt[3]{q}}$ & 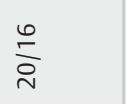 & $\frac{\infty}{\gamma}$ & $\underset{\substack{\infty \\
i n}}{\stackrel{\nabla}{\infty}}$ & $\frac{\stackrel{n}{N}}{m}$ & 1 & $\frac{\pi}{\frac{\pi}{N}}$ \\
\hline बू & 气 & 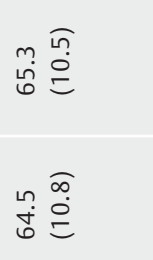 & $\begin{array}{l}\widehat{T} \\
0 \\
1 \\
0 \\
0 \\
0 \\
0\end{array}$ & 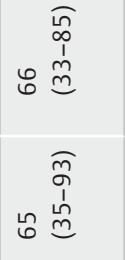 & $\begin{array}{l}\text { co } \\
0 \\
1 \\
0 \\
0 \\
0\end{array}$ & $\begin{array}{l}\widehat{a} \\
\infty \\
1 \\
\omega \\
\\
6\end{array}$ & 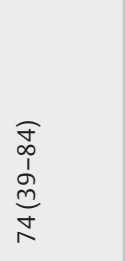 & 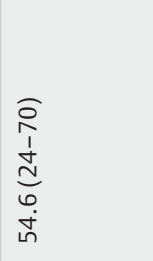 & $\begin{array}{l}\text { ạ } \\
\stackrel{\dot{E}}{\bar{\sigma}} \\
\text { in }\end{array}$ & 1 & 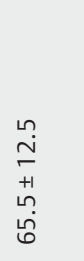 \\
\hline \multicolumn{2}{|c|}{ 完 } & $\stackrel{?}{\stackrel{2}{r}}$ & in & ర్ల & $\hat{6}$ & $\stackrel{m}{m}$ & $\simeq$ & $\stackrel{N}{N}$ & $\stackrel{\infty}{n}$ & 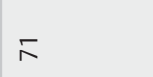 & $\stackrel{m}{q}$ \\
\hline \multicolumn{2}{|c|}{ 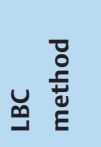 } & 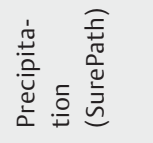 & 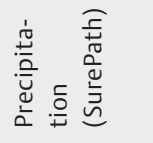 & 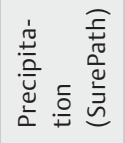 & 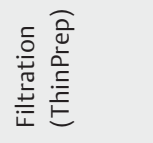 & 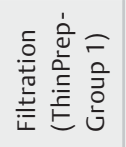 & 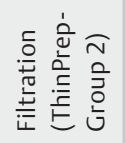 & 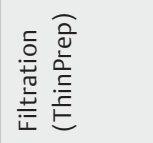 & 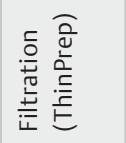 & 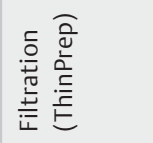 & 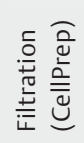 \\
\hline 峛 & $\frac{2}{x}$ & $z$ & $z$ & $z$ & $z$ & $z$ & $z$ & $z$ & $z$ & $z$ & $z$ \\
\hline$\frac{\overline{5}}{\overline{\bar{y}}}$ & & 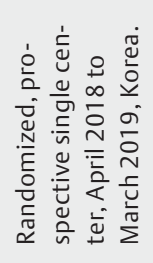 & 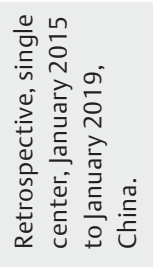 & 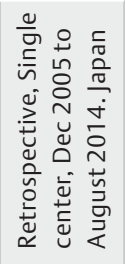 & 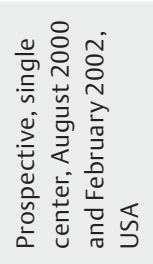 & 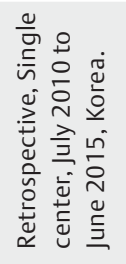 & & 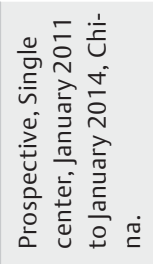 & 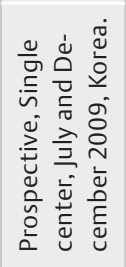 & 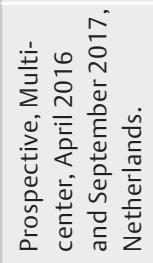 & 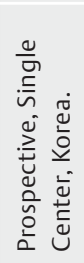 \\
\hline $\begin{array}{l}\text { D̀ } \\
\text { 章 } \\
\end{array}$ & & 胥空 & 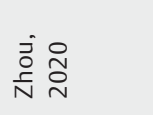 & 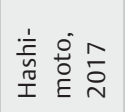 & 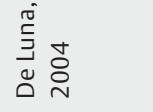 & 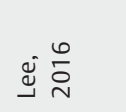 & & 言 & פ̃ $\bar{N}$ & 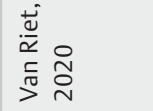 & 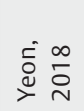 \\
\hline
\end{tabular}




\begin{tabular}{|c|c|c|c|c|c|c|c|c|c|c|c|}
\hline 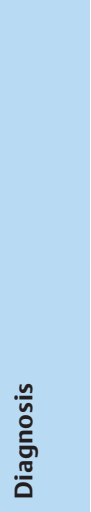 & 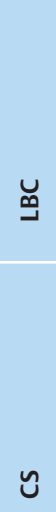 & 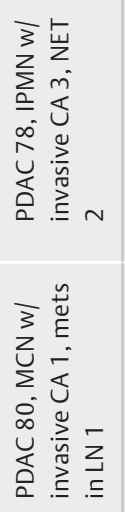 & 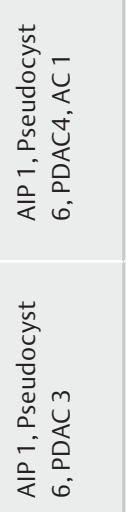 & 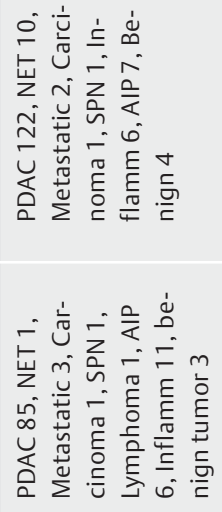 & 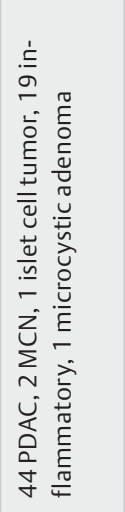 & 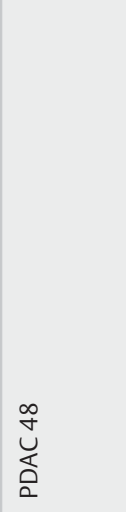 & & 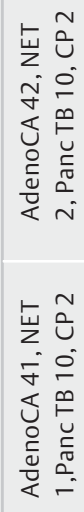 & 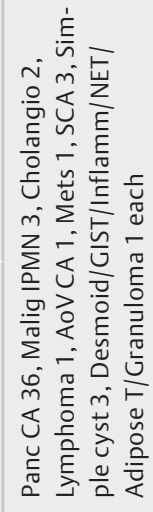 & 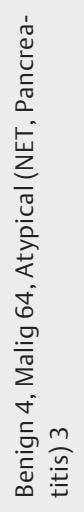 & $\frac{\alpha}{z}$ \\
\hline 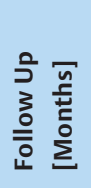 & & E & E & $\frac{a}{z}$ & $\begin{array}{l}\widehat{E} \\
\stackrel{E}{N} \\
\infty \\
\infty \\
E \\
m\end{array}$ & $\stackrel{E}{\sim}$ & & 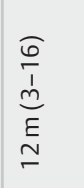 & $\frac{\alpha}{z}$ & $\stackrel{E}{\sim}$ & E \\
\hline 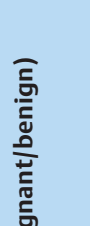 & $\stackrel{M}{9}$ & 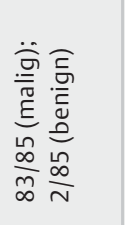 & 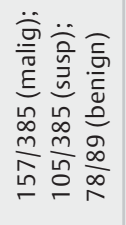 & 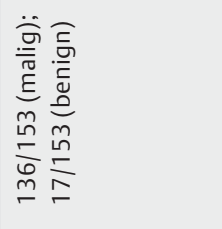 & 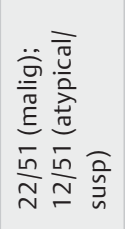 & 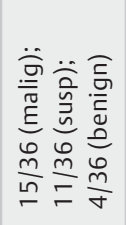 & 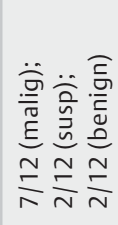 & 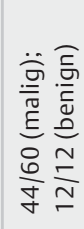 & 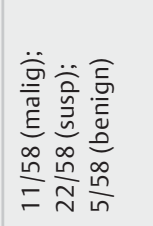 & 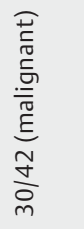 & 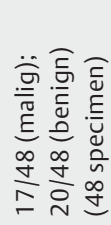 \\
\hline 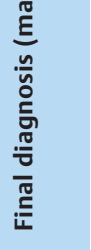 & 气 & 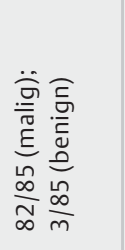 & 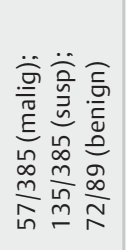 & 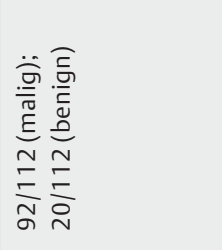 & 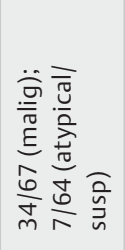 & 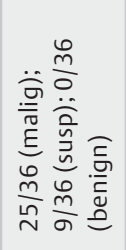 & 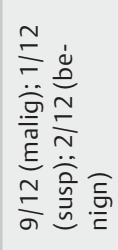 & 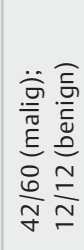 & 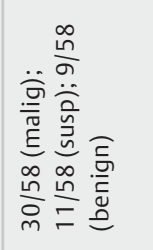 & 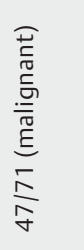 & 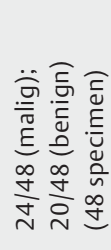 \\
\hline & 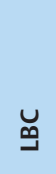 & 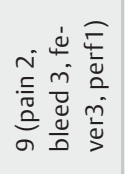 & $\frac{c}{z}$ & $\frac{u}{z}$ & 1 & 1 & 1 & 1 & & & 1 \\
\hline 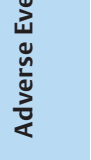 & 气 & 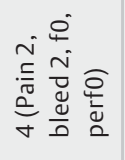 & $\frac{a}{z}$ & $\frac{\mathrm{v}}{z}$ & 1 & 1 & 1 & 1 & 童 & $\frac{\tilde{\sigma}}{2}$ & 1 \\
\hline 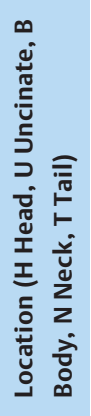 & 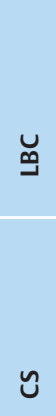 & 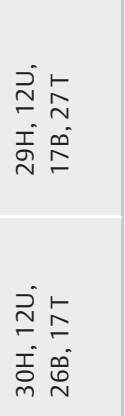 & 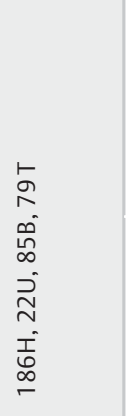 & 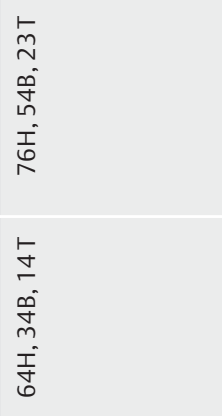 & 1 & 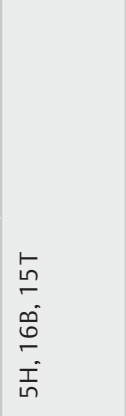 & 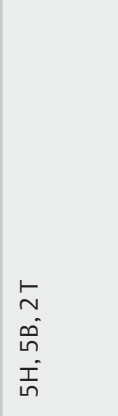 & 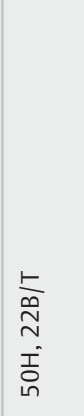 & 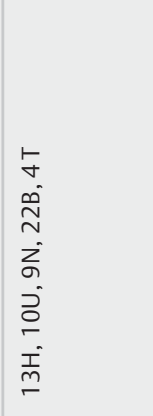 & 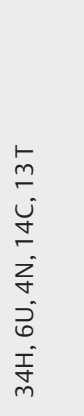 & 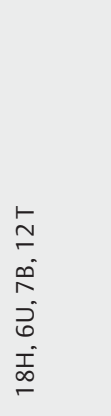 \\
\hline 高 & & 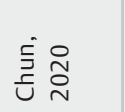 & $\begin{array}{l}z \\
\frac{\Xi}{N} \\
\frac{1}{N}\end{array}$ & 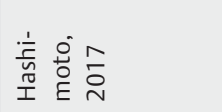 & 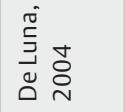 & \multicolumn{2}{|l|}{ פ্் } & $\stackrel{\bar{\partial}}{\sigma} \stackrel{\sigma}{N}$ & 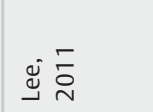 & 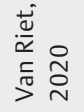 & 竞 $\stackrel{\infty}{\stackrel{\infty}{\nu}}$ \\
\hline
\end{tabular}


> Table2 Pooled performance of CS and LBC techniques.

\begin{tabular}{|c|c|c|c|}
\hline & Malignant & Benign & Suspicious \\
\hline \multicolumn{4}{|l|}{ Pooled odds ratio ( $95 \%$ confidence interval) } \\
\hline Conventional smear vs Liquid based cytology (LBC) & NA & $\begin{array}{l}\mathrm{OR}=1.1(0.7-1.75) \\
P=0.7 \\
\mathrm{I}^{2}=2 ; 8 \text { studies }\end{array}$ & $\begin{array}{l}\mathrm{OR}=1.23(0.96-1.59) \\
P=0.1 \\
\mathrm{I}^{2}=76 ; 5 \text { studies }\end{array}$ \\
\hline Conventional smear vs Precipitation-LBC (SurePath) & $\begin{array}{l}\mathrm{OR}=0.39(0.19-0.8) \\
P=0.01 \\
\mathrm{I}^{2}=60 ; 3 \text { studies }\end{array}$ & $\begin{array}{l}\mathrm{OR}=1.1(0.6-2.1) \\
P=0.7 \\
\mathrm{I}^{2}=48 ; 3 \text { studies }\end{array}$ & $-\mathrm{NA}-$ \\
\hline $\begin{array}{l}\text { Conventional smear vs Filtration-LBC } \\
\text { (ThinPrep, CellPrep) }\end{array}$ & $\begin{array}{l}\mathrm{OR}=1.69(1.02-2.79) \\
P=0.04 \\
I^{2}=56 ; 7 \text { studies }\end{array}$ & $\begin{array}{l}\mathrm{OR}=1.1(0.54-2.17) \\
P=0.8 \\
\mathrm{I}^{2}=0 ; 5 \text { studies }\end{array}$ & $\begin{array}{l}\mathrm{OR}=0.47(0.27-0.8) \\
P=0.006 \\
\mathrm{I}^{2}=0 ; 4 \text { studies }\end{array}$ \\
\hline \multicolumn{4}{|l|}{ Pooled proportions ( $95 \%$ confidence interval) } \\
\hline Conventional smear & $\begin{array}{l}64.9 \%(43.4-81.7) \\
I^{2}=96 ; 10 \text { studies }\end{array}$ & $\begin{array}{l}26.4 \%(8.2-58.8) \\
\mathrm{I}^{2}=94 ; 8 \text { studies }\end{array}$ & $\begin{array}{l}22.8 \%(10.4-42.7) \\
I^{2}=93 ; 5 \text { studies }\end{array}$ \\
\hline \multicolumn{4}{|l|}{ Liquid-based cytology subtypes } \\
\hline Precipitation-LBC (SurePath) & $\begin{array}{l}84.1 \%(50.9-96.4) \\
\mathrm{I}^{2}=98 ; 3 \text { studies }\end{array}$ & $\begin{array}{l}23.2 \%(3.3-72.5) \\
I^{2}=98 ; 3 \text { studies }\end{array}$ & NA \\
\hline Filtration-LBC (ThinPrep) & $\begin{array}{l}48.6 \%(24.2-73.7) \\
I^{2}=84 ; 7 \text { studies }\end{array}$ & $\begin{array}{l}29.1 \%(6.7-70.3) \\
I^{2}=85 ; 5 \text { studies }\end{array}$ & $\begin{array}{l}27.5 \%(11.9-51.5) \\
I^{2}=18 ; 4 \text { studies }\end{array}$ \\
\hline
\end{tabular}

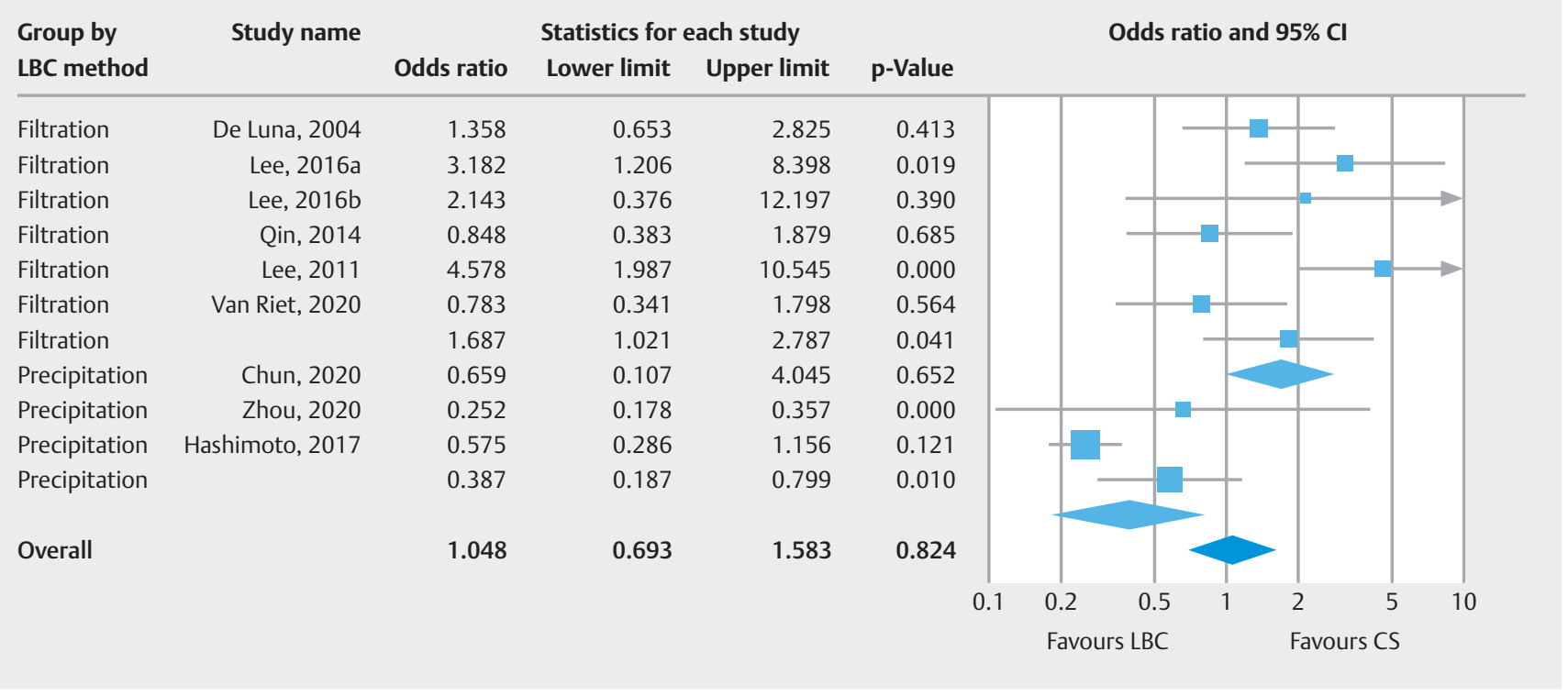

- Fig. 1 OR malignant.

b) CS cytology performed significantly better than filtrationbased LBC technique in diagnosing suspicious, and malignant pancreatic lesions $[\mathrm{OR}=1.69(1.02-2.79) ; P=0.04$, OR $=0.47(0.27-0.8) ; P=0.006]$. For benign lesions, outcomes of the two techniques were comparable.

c) Precipitation-based LBC performed significantly better than CS cytology in diagnosing malignant pancreatic lesions [OR $=0.39(0.19-0.8) ; P=0.01]$, but this effect was not seen with respect to benign lesions.
Pooled rates of performance in terms of final diagnosis (malignant, suspicious or benign) of CS cytology and LBC techniques and pooled odds are summarized in $>$ Table 2 and $>$ Fig. 1, > Fig. 2, > Fig. 3, > Fig. 4, > Fig. 5 and > Fig. 6.

2. Pooled diagnostic performance of CS, Precipitation-based and Filtration-based LBC techniques

Precipitation-based LBC had higher pooled accuracy (85.2\%) and sensitivity (83.6\%) compared to CS (79.7\% and $79.2 \%$, respectively) and filtration-based LBC (77.3\% and $68.3 \%$, 


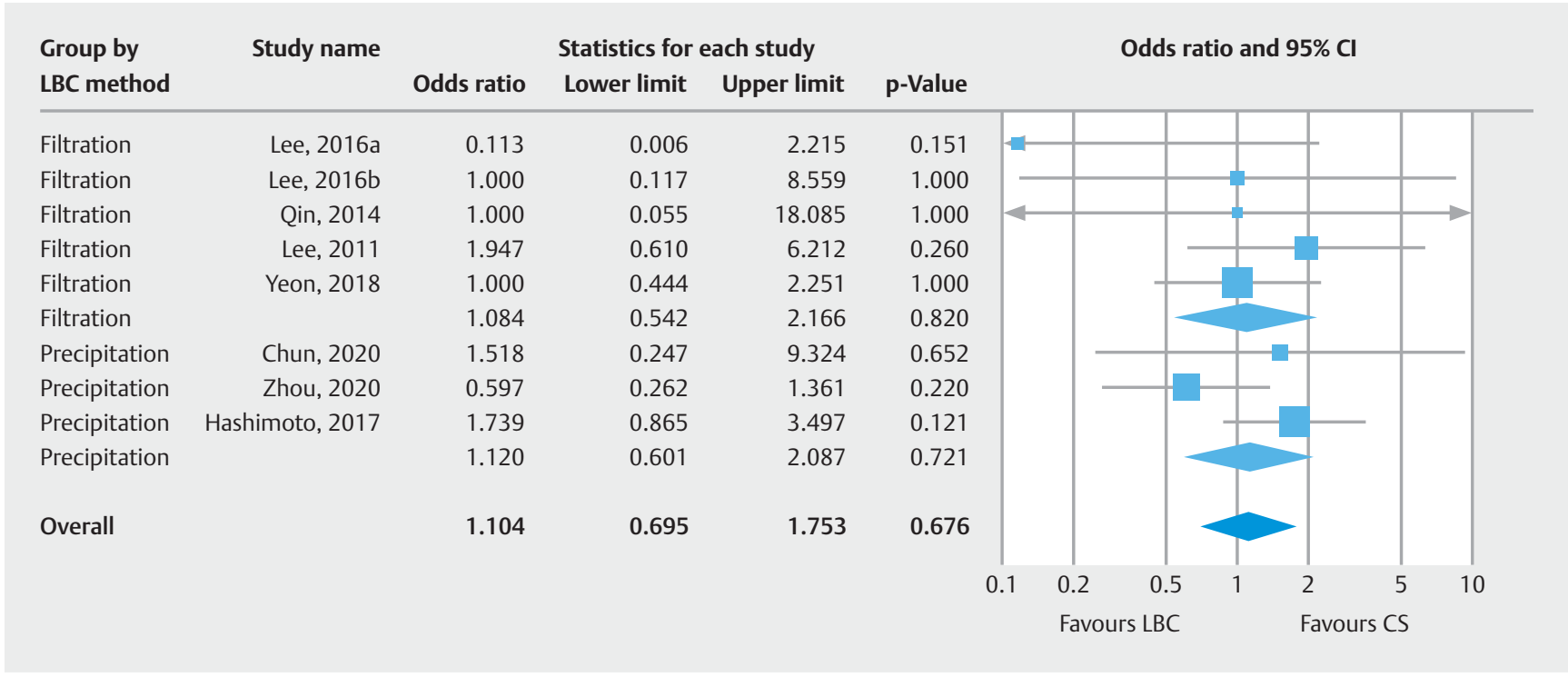

Fig. 2 OR benign.

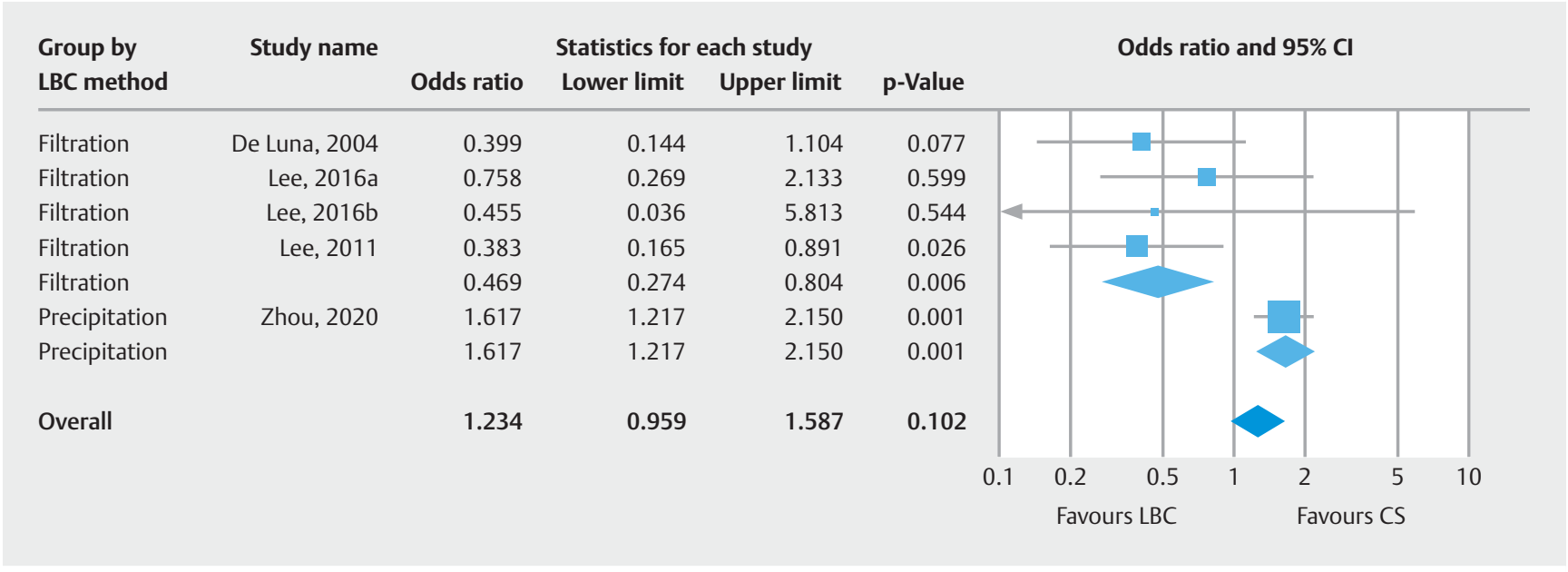

- Fig. 3 OR suspicious.

respectively). Specificity and PPV were comparable in all three techniques. NPV was found to be higher with filtration-based LBC technique (50.9\%) as compared with CS (46.2\%) and precipitation-based LBC techniques (35.4\%). Details are summarized in $>$ Table 3 and Supplementary Fig. 1 to 5

\section{Validation of meta-analysis results}

\section{Sensitivity analysis}

To assess whether any one study had a dominant effect on the meta-analysis, we excluded one study at a time and analyzed its effect on the main summary estimate. In the pooled OR analysis, we noted a significant change to the pooled rates for CS vs LBC by precipitation, when the studies by Lee et al 2011 and Yeon et al 2018 were removed (pooled $O R=1.4, P=0.1$ and pooled $O R=1.7, P=0.1$; respectively). No changes to the final pooled rates were noted in the analysis of benign and/or suspi- cious lesions. In the analysis of proportions, we did not notice any changes to the pooled rates when any one study was removed at a time in the diagnosis of any of the lesions.

\section{Heterogeneity}

We assessed dispersion of the calculated rates using the $\mathrm{Cl}$ and $\mathrm{I}^{2}$ percentage values. The $\mathrm{Cl}$ gives an idea of the range of the dispersion and $\mathrm{I}^{2}$ tell us what proportion of the dispersion is true vs chance [29]. Overall, a considerable to substantial degree of heterogeneity was noted in the analysis. Based on our analysis, the types of LBC method (precipitation, filtration), brand of LBC medium (SurePath ${ }^{\mathrm{TM}}$, ThinPrep ${ }^{\circledR}$, CellPrep ${ }^{\circledR}$ ) and final diagnosis of the lesion (benign, suspicious, or malignant) seemed to explain the observed heterogeneity to some extent. Other possible variables that might have contributed to the heterogeneity, which were not amenable to analysis, were the number of needle passes performed, FNA needle gauge used 


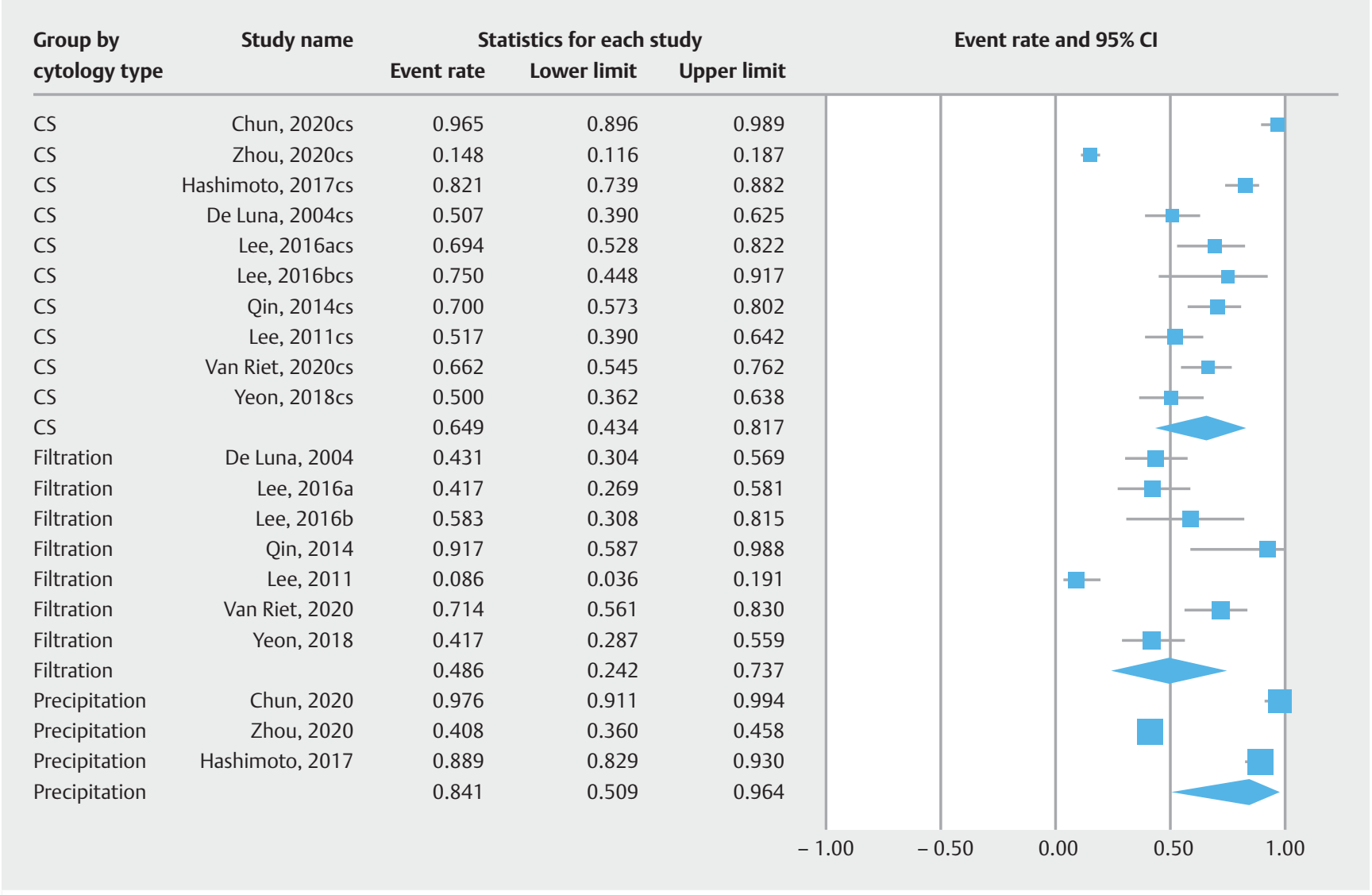

Fig. 4 PR malignant.

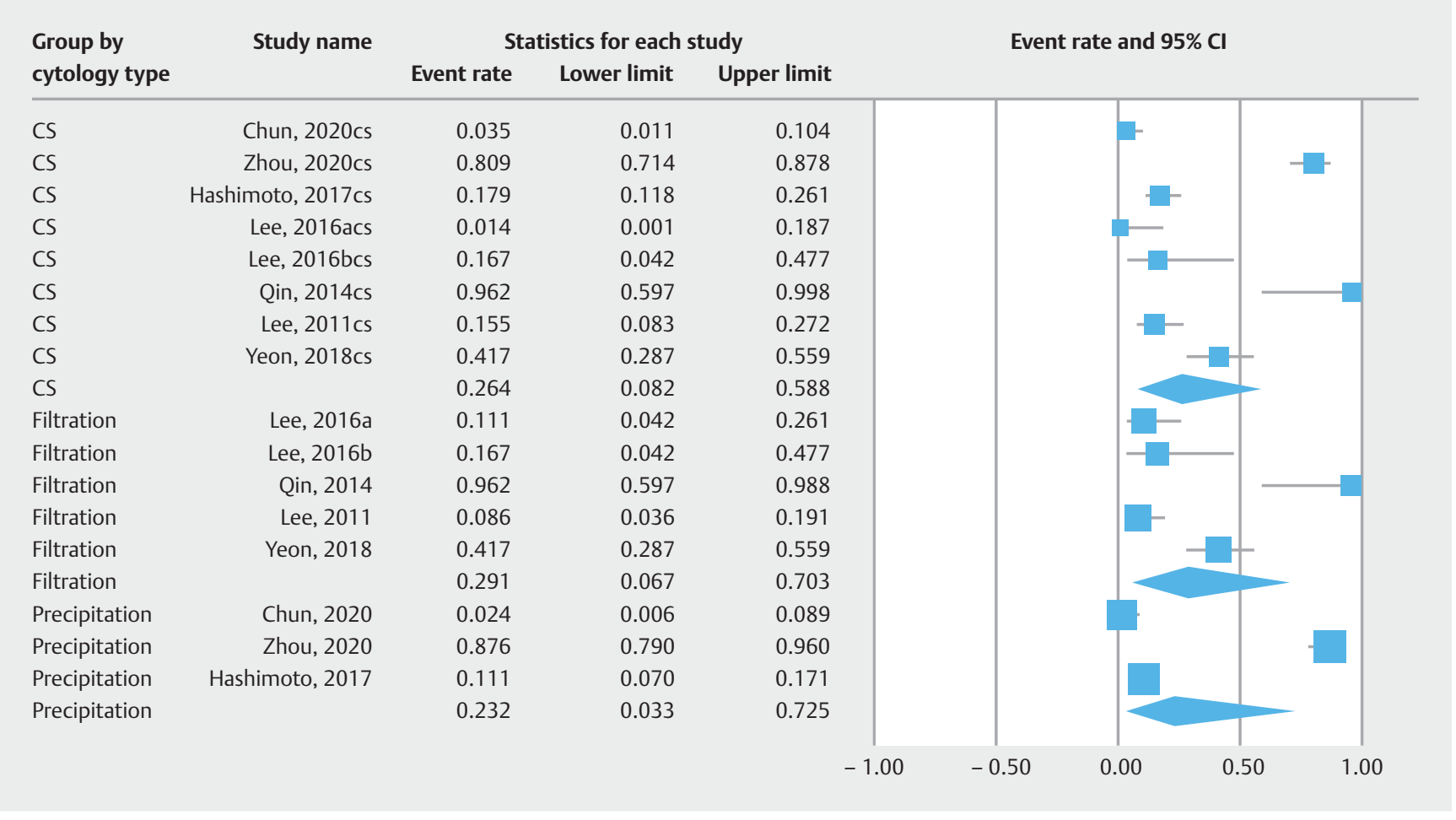

Fig. 5 PR benign. 


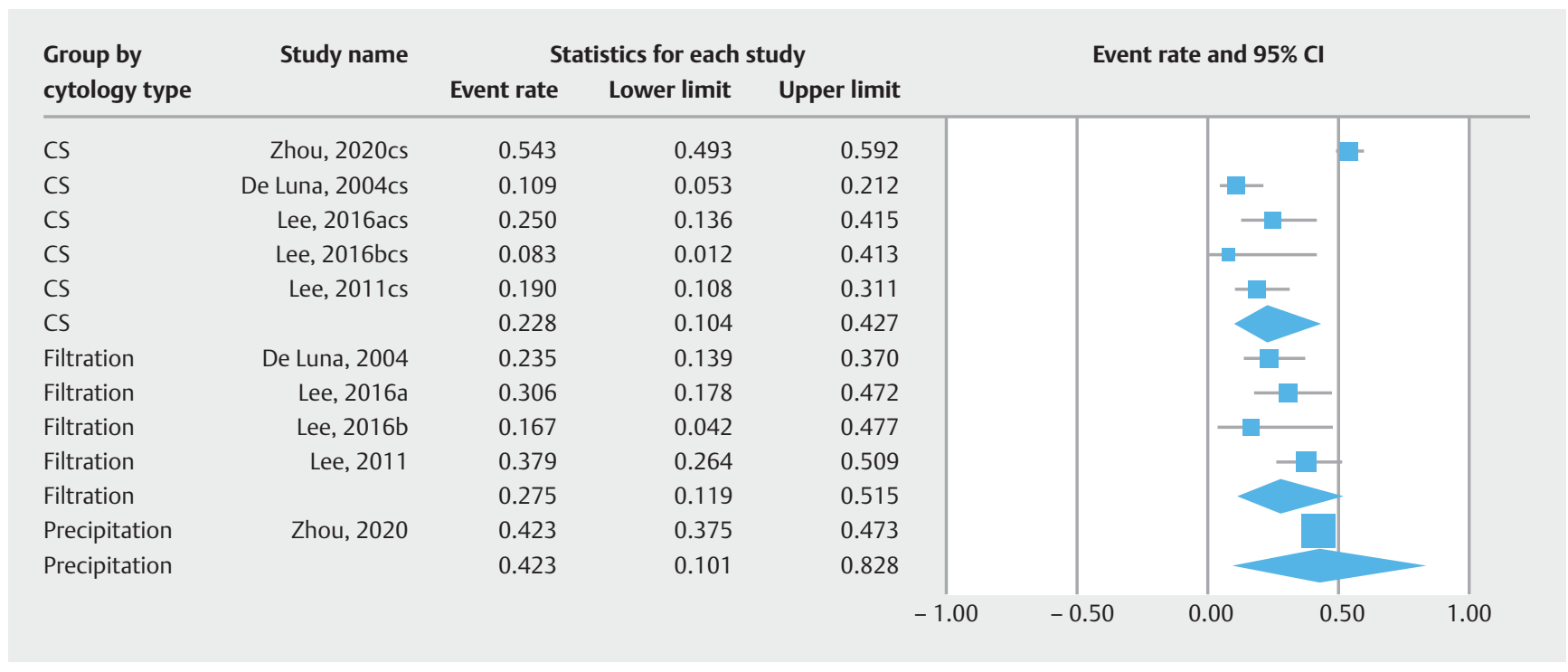

Fig. 6 PR suspicious.

- Table 3 Pooled accuracy/sensitivity/specificity/PPV/NPV of techniques.

\begin{tabular}{|c|c|c|c|c|c|}
\hline & Accuracy & Sensitivity & Specificity & PPV & NPV \\
\hline CS & $\begin{array}{l}79.7 \%(72.2-85.6) \\
I^{2}=86 \\
8 \text { studies }\end{array}$ & $\begin{array}{l}79.2 \%(70.7-85.7) \\
\mathrm{I}^{2}=89 \\
8 \text { studies }\end{array}$ & $\begin{array}{l}99.4 \%(98.4-99.8) \\
I^{2}=0 \\
7 \text { studies }\end{array}$ & $\begin{array}{l}99.5 \%(98.5-99.8) \\
\mathrm{I}^{2}=0 \\
6 \text { studies }\end{array}$ & $\begin{array}{l}46.2 \%(27.2-66.4) \\
I^{2}=96 ; \\
6 \text { studies }\end{array}$ \\
\hline \multicolumn{6}{|c|}{ Liquid based cytology sub-types } \\
\hline $\begin{array}{l}\text { Precipitation-LBC } \\
\text { (SurePath) }\end{array}$ & $\begin{array}{l}85.2 \%(74.4-91.9) \\
I^{2}=76 \\
3 \text { studies }\end{array}$ & $\begin{array}{l}83.6 \%(70.7-91.5) \\
I^{2}=85 \\
3 \text { studies }\end{array}$ & $\begin{array}{l}99.5 \%(97.6-99.9) \\
1^{2}=0 \\
3 \text { studies }\end{array}$ & $\begin{array}{l}99.5 \%(97.6-99.9) \\
I^{2}=0 \\
3 \text { studies }\end{array}$ & $\begin{array}{l}35.4 \%(14.5-63.9) \\
I^{2}=91 ; \\
3 \text { studies }\end{array}$ \\
\hline Filtration-LBC (ThinPrep) & $\begin{array}{l}77.3 \%(67.1-85) \\
1^{2}=49 ; \\
5 \text { studies }\end{array}$ & $\begin{array}{l}68.3 \%(55.3-79) \\
1^{2}=64 \\
5 \text { studies }\end{array}$ & $\begin{array}{l}99.5 \%(97.6-99.9) \\
1^{2}=0 \\
4 \text { studies }\end{array}$ & $\begin{array}{l}99.5 \%(97.6-99.9) \\
I^{2}=0 \\
3 \text { studies }\end{array}$ & $\begin{array}{l}50.9 \%(24.4-76.9) \\
I^{2}=92 \\
3 \text { studies }\end{array}$ \\
\hline
\end{tabular}

CS, conventional smear; LBC, liquid-based cytology; PPV, positive predictive value; NPV, negative predictive value.

(19G, 22G, 25G), location of pancreatic lesion (head, uncinate, body, tail) and study design (retrospective, prospective). Overall, considerable heterogeneity was noted in the analysis.

\section{Publication bias}

Publication bias was not evaluated as the number of studies in our analysis was less than 10 .

\section{Discussion}

We found that EUS-guided FNA using conventional smear (CS) cytology was superior to filtration-based LBC for diagnosing malignant as well as suspicious pancreatic lesions. On the other hand, precipitation-based LBC was superior to CS cytology, in particular, for diagnosing malignant pancreatic lesions. We found no significant difference in the diagnostic odds of the three techniques in terms of diagnosing benign pancreatic lesions.
In terms of overall diagnostic performance, EUS-guided fineneedle aspiration using precipitation-based liquid cytology technique had higher accuracy and sensitivity compared to CS and filtration-based liquid cytology techniques $(85.2 \%$ and $83.6 \%$, respectively).

EUS-guided FNA is a safe, cost-effective and widely accepted method for evaluating and sampling solid pancreatic lesions [41]. For decades, the CS technique has been used and has demonstrated a diagnostic sensitivity for pancreatic malignancy as high as $96 \%[42,43]$. CS involves the FNA sample being smeared directly onto a glass slide and then either air-dried for Diff-quick staining or wet-fixed with an ethanol-based fixative for Papanicolaou staining, although other stains can be employed.

There are some important differences between CS preparations, filtration-based LBC, and precipitation-based LBC that deserve a mention. CS preparations have the advantage of the pathologist knowing that the cellular material on the slide represents a single pass (meaning that increased cellularity has 
- Table4 Advantages and disadvantages of CS and LBC techniques.

\begin{tabular}{|c|c|c|c|}
\hline & Conventional Smear (CS) & LBC (Filtration) & LBC (Precipitation) \\
\hline \multirow[t]{4}{*}{ Advantages } & Better gauge of cellularity & No ROSE needed & No ROSE needed \\
\hline & Preserved extracellular material & Single slide & Single slide \\
\hline & Preserved cytologic architecture & \multirow[t]{2}{*}{ Flatter cell distribution } & \multirow[t]{2}{*}{ Improved satisfactory rate } \\
\hline & Stain options & & \\
\hline \multirow[t]{2}{*}{ Disadvantages } & Requires ROSE & Increased unsatisfactory rate & More cell crowding/overlap \\
\hline & More slides to evaluate & $\begin{array}{l}\text { Loss of cellularity gauge, extracellular } \\
\text { material, cytologic architecture, and stain } \\
\text { options }\end{array}$ & $\begin{array}{l}\text { Loss of cellularity gauge, extracellular } \\
\text { material, cytologic architecture, and stain } \\
\text { options }\end{array}$ \\
\hline
\end{tabular}

more meaning and can better support an abnormality). On the other hand, when LBC is used, often several passes are put into the same vial, and therefore, cellularity becomes less informative. CS also better preserves extracellular material that may be present in a lesion (eg, hyalinization that occurs in SPT). In addition, it is generally understood that CS allows better evaluation of cytomorphologic architecture; for example, loss of cohesion in carcinoma cells is often more evident on a CS. Finally, it is helpful to have the option for different staining methods (Diff-Quik ${ }^{\mathrm{TM}}$ ) and Papanicolaou stains with CS.

The major advantage of CS is the needed availability of a ROSE where a cytopathologist is present during the procedure. The rationale for using ROSE in EUS-FNA tissue acquisition is that it allows real-time evaluation of sample adequacy and diagnostic yield. Additionally, on-site cytopathologists can determine whether additional sampling is required for further diagnosis or if specimen triage is necessary for additional studies (eg, immunohistochemistry or flow cytometry) [44,45]. Despite its proven advantage $[46,47]$, ROSE is not readily available in all healthcare centers across the world. According to a study on practice patterns in EUS-FNA published in 2016, ROSE was available in only $48 \%$ of European and $55 \%$ of Asian centers, but in almost all centers (98\%) in the United States. The obstacles to expanding ROSE include limited cytopathologist staffing, cost-effective performance, and longer procedural duration [48].

LBC was first introduced in 1991 and has been used extensively in the analysis of fine-needle aspirations of the thyroid [49-51] and cervical cytology [52-54]. Although tissue architecture and the determination of cellularity can be made more difficult on LBC as compared to CS, LBC does allow for a reduction in artifacts related to sample drying and elimination of blood contamination by lysing red blood cells thereby resulting in a cleaner background. In addition, because the cellular elements are within a circle in the center of the slide, it can be less time-consuming for the cytopathologist compared with CS where the samples are irregularly distributed over multiple glass slides $[18,55]$.

Filtration-based and precipitation-based are two types of LBC methods. They have some similarities and few subtle differences. In general, the filtration method has flatter cell layer dis- tribution, which is considered its main advantage over the precipitation method as cells can be easier to visualize over a larger diameter area. The filtration method does suffer from the filter itself getting clogged by interfering material (such as blood or mucus) that increases the number of unsatisfactory results as compared to the precipitation method. The nuclear features of malignancy or benignity should be similarly evident in both LBC preparations. Both methods have the advantage of being a single slide for evaluation, compared to CS which usually has multiple slides per case [56]. Differences between the preparation types outlined in $>$ Table 4.

The strengths of this review are as follows: systematic literature search with well-defined inclusion criteria, careful exclusion of redundant studies, inclusion of good quality studies with detailed extraction of data and rigorous evaluation of study quality. Our study included only those studies in which a comparative analysis between CS and LBC was performed in pancreatic lesions. We excluded studies in which either only one technique was evaluated $[57,58]$ or non-pancreatic lesions were evaluated $[59,60]$ There are limitations to this study as well, most of which are inherent in any meta-analysis. We were unable to evaluate the effect of lesion location, size, number of passes, needle size, or operator experience on our results. We included studies that were retrospective in nature contributing to selection bias and our analysis has the limitation of non-causal comparison with significant heterogeneity. While a majority of the lesions diagnosed in our study were pancreatic adenocarcinomas, we were unable to evaluate the efficacy of either cytology techniques for other malignant and benign pancreatic etiologies given their underrepresentation in included studies. In one of the included studies, atypical and suspicious lesions were grouped together [35]. Finally, our analysis included only three studies that used the precipitation-based LBC technique, which may have influenced our results.

\section{Conclusion}

Our study is the first in the literature to evaluate the diagnostic performance of conventional smear in the absence of ROSE with LBC in EUS-guided FNA sampling of solid pancreatic lesions. We found that CS and LBC techniques have comparable efficacy in 
terms of characterizing pancreatic lesions as either malignant, suspicious or benign. However, given the differences in preparation methods of LBC, comparative analysis with CS is inaccurate. Hence, we decided to compare CS with both LBC techniques and found that precipitation-based LBC (SurePath) outperforms CS cytology without ROSE for diagnosing malignant pancreatic lesions. However, CS cytology outperforms the filtration-based LBC (ThinPrep, CellPrep) technique in diagnosing both malignant and suspicious pancreatic lesions. In conclusion, in the absence of ROSE, precipitation-based LBC should be preferred over CS technique in EUS-guided sampling of pancreatic lesions. Finally, regardless of the cytologic preparation method, EUS-FNA cytology continues to remain a useful and accurate diagnostic tool for pancreatic lesions.

\section{Acknowledgement}

The authors thank Dana Gerberi, MLIS, Librarian, Mayo Clinic Libraries, for her help with the systematic literature search.

\section{Competing interests}

Dr. Adler is a consultant for Boston Scientific.

\section{References}

[1] Tharian B, Tsiopoulos F, George $\mathrm{N}$ et al. Endoscopic ultrasound fine needle aspiration: Technique and applications in clinical practice. World J Gastrointest Endosc 2012; 4: 532-544

[2] Weston BR, Bhutani MS. Optimizing diagnostic yield for EUS-guided sampling of solid pancreatic lesions: a technical review. Gastroenterol Hepatol (NY) 2013; 9: 352-363

[3] Santo E, Bar-Yishay I. Pancreatic solid incidentalomas. Endosc Ultrasound 2017; 6: S99-S103

[4] Bray F, Ferlay J, Soerjomataram I et al. Global cancer statistics 2018: GLOBOCAN estimates of incidence and mortality worldwide for 36 cancers in 185 countries. CA Cancer J Clin 2018; 68: 394-424

[5] Ferlay J, Ervik M, Lam F et al. Global cancer Observatory: cancer today. Lyon, France: International agency for research on cancer, Cancer Today; 2018

[6] DeWitt J, McGreevy K, Sherman S et al. Utility of a repeated EUS at a tertiary-referral center. Gastrointest Endosc 2008; 67: 610-619

[7] Iglesias-Garcia J, Dominguez-Munoz E, Lozano-Leon A et al. Impact of endoscopic ultrasound-guided fine needle biopsy for diagnosis of pancreatic masses. World J Gastroentero 2007; 13: 289

[8] Bang JY, Hebert-Magee S, Trevino J et al. Randomized trial comparing the 22-gauge aspiration and 22-gauge biopsy needles for EUS-guided sampling of solid pancreatic mass lesions. Gastrointest Endosc 2012; 76: $321-327$

[9] Hucl T, Wee E, Anuradha $S$ et al. Feasibility and efficiency of a new $22 \mathrm{G}$ core needle: a prospective comparison study. Endoscopy 2013; 45: 792-798

[10] Berzosa M, Villa N, El-Serag HB et al. Comparison of endoscopic ultrasound guided 22-gauge core needle with standard 25-gauge fineneedle aspiration for diagnosing solid pancreatic lesions. Endosc UItrasound 2015; 4: 28

[11] Syed A, Babich O, Rao B et al. Endoscopic ultrasound guided fineneedle aspiration vs core needle biopsy for solid pancreatic lesions:
Comparison of diagnostic accuracy and procedural efficiency. Diagnostic cytopathology 2019; 47: 1138-1144

[12] Sweeney J, Soong L, Goyal A. Endoscopic ultrasound-guided tissue acquisition of solid mass lesions of the pancreas: A retrospective comparison study of fine-needle aspiration and fine-needle biopsy. Diagn Cytopathol 2020; 48: 322-329

[13] Petrone MC, Arcidiacono PG. Basic technique in endoscopic ultrasound-guided fine needle aspiration for solid lesions: How many passes? Endosc Ultrasound 2014; 3: 22-27

[14] Matynia AP, Schmidt RL, Barraza G et al. Impact of rapid on-site evaluation on the adequacy of endoscopic-ultrasound guided fine-needle aspiration of solid pancreatic lesions: a systematic review and metaanalysis. J Gastroenterol Hepatol 2014; 29: 697-705

[15] Hewitt MJ, McPhail MJ, Possamai L et al. EUS-guided FNA for diagnosis of solid pancreatic neoplasms: a meta-analysis. Gastrointest Endosc 2012; 75: 319-331

[16] Haba S, Yamao K, Bhatia V et al. Diagnostic ability and factors affecting accuracy of endoscopic ultrasound-guided fine needle aspiration for pancreatic solid lesions: Japanese large single center experience. J Gastroenterol 2013; 48: 973-981

[17] da Cunha Santos G, Saieg MA. Preanalytic specimen triage: Smears, cell blocks, cytospin preparations, transport media, and cytobanking. Cancer Cytopathol 2017; 125: 455-464

[18] Yeon MH, Jeong HS, Lee HS et al. Comparison of liquid-based cytology (CellPrepPlus) and conventional smears in pancreaticobiliary disease. Korean J Intern Med 2018; 33: 883-892

[19] Bernstein SJ, Sanchez-Ramos L, Ndubisi B. Liquid-based cervical cytologic smear study and conventional Papanicolaou smears: a metaanalysis of prospective studies comparing cytologic diagnosis and sample adequacy. Am J Obstet Gynecol 2001; 185: 308-317

[20] Meara RS, Jhala D, Eloubeidi MA et al. Endoscopic ultrasound-guided FNA biopsy of bile duct and gallbladder: analysis of 53 cases. Cytopathology 2006; 17: 42-49

[21] Stroup DF, Berlin JA, Morton SC et al. Meta-analysis of observational studies in epidemiology: a proposal for reporting. Meta-analysis Of Observational Studies in Epidemiology (MOOSE) group. JAMA 2000; 283: 2008-2012

[22] Moher D, Liberati A, Tetzlaff J et al. Preferred reporting items for systematic reviews and meta-analyses: The prisma statement. Ann Intern Med 2009; 151: 264-269

[23] Lee JK, Choi ER, Jang TH et al. A prospective comparison of liquidbased cytology and traditional smear cytology in pancreatic endoscopic ultrasound-guided fine needle aspiration. Acta Cytologica 2011; 55: 401-407

[24] Lee KJ, Kang YS, Cho MY et al. Comparison of cytologic preparation methods in endoscopic ultrasound-guided fine needle aspiration for diagnosis of pancreatic adenocarcinoma. Pancreatology 2016; 16: 824-828

[25] Stang A. Critical evaluation of the Newcastle-Ottawa scale for the assessment of the quality of nonrandomized studies in meta-analyses. Eur J Epidemiol 2010; 25: 603-605

[26] DerSimonian R, Laird N. Meta-analysis in clinical trials. Control Clin Trials 1986; 7: 177-188

[27] Sutton AJ AK, Jones DR et al. Methods for meta-analysis in medical research. New York: J. Wiley; 2000

[28] Higgins JP, Thompson SG, Deeks JJ et al. Measuring inconsistency in meta-analyses. BMJ 2003; 327: 557-560

[29] Mohan BP, Adler DG. Heterogeneity in systematic review and metaanalysis: how to read between the numbers. Gastrointest Endosc 2019; 89: 902-903

[30] Duval S, Tweedie R. Trim and Fill: a simple funnel-plot-based method of testing and adjusting for publication bias in meta-analysis. Biometrics 2000; 56: 455-463 
[31] Rothstein HR, Sutton A], Borenstein M. Publication bias in meta-analysis: Prevention, assessment and adjustments. John Wiley \& Sons; 2006

[32] Chun JW, Lee K, Lee SH et al. Comparison of liquid-based cytology with conventional smear cytology for EUS-guided FNA of solid pancreatic masses: a prospective randomized noninferiority study. Gastrointest Endosc 2020; 91: 837-846.e831

[33] Zhou W, Gao L, Wang S-M et al. Comparison of smear cytology and liquid-based cytology in EUS-guided fine-needle aspiration of pancreatic lesion: experience from a large tertiary center. Gastrointest Endosc 2020; 91: 932-942

[34] Hashimoto S, Taguchi H, Higashi M et al. Diagnostic efficacy of liquidbased cytology for solid pancreatic lesion samples obtained with endoscopic ultrasound-guided fine-needle aspiration: Propensity score-matched analysis. Digest Endosc 2017; 29: 608-616

[35] de Luna R, Eloubeidi MA, Sheffield MV et al. Comparison of ThinPrep and conventional preparations in pancreatic fine-needle aspiration biopsy. Diagnostic Cytopathology 2004; 30: 71-76

[36] Lee YS, Son JH, Kim NH et al. Diagnostic efficacy of liquid-based cytology in endoscopic ultrasoundguided fine-needle aspiration for solid pancreas lesion: Single academic institution experience. Pancreatology 2018; 18: S42

[37] Qin SY, Zhou Y, Li P et al. Diagnostic efficacy of cell block immunohistochemistry, smear cytology, and liquid-based cytology in endoscopic ultrasound-guided fine-needle aspiration of pancreatic lesions: a single-institution experience. PLoS ONE 2014; 9: e108762

[38] Lee JK, Choi ER, Jang TH et al. A prospective comparison of liquidbased cytology and traditional smear cytology in pancreatic endoscopic ultrasound-guided fine needle aspiration. Acta Cytol 2011; 55 : 401-407

[39] van Riet PA, Quispel R, Cahen DL et al. Diagnostic yield and agreement on fine-needle specimens from solid pancreatic lesions : comparing the smear technique to liquid-based cytology. Endosc Int Open 2020; 8: E155-E162

[40] Qin SY, Jiang HX, Zhang XL et al. Evaluation of endoscopic ultrasoundguided fine-needle aspiration biopsy of cell block with immunostaining for panceatic lesions. J Digest Dis 2014; 1: 109

[41] Uehara H, Ikezawa K, Kawada N et al. Diagnostic accuracy of endoscopic ultrasound-guided fine needle aspiration for suspected pancreatic malignancy in relation to the size of lesions. J Gastroenterol Hepatol 2011; 26: 1256-1261

[42] Iglesias-Garcia J, Lariño-Noia J, Abdulkader I et al. Rapid on-site evaluation of endoscopic-ultrasound-guided fine-needle aspiration diagnosis of pancreatic masses. World J Gastroenterol 2014; 20: 94519457

[43] Iglesias-Garcia J, Dominguez-Munoz JE, Abdulkader I et al. Influence of on-site cytopathology evaluation on the diagnostic accuracy of endoscopic ultrasound-guided fine needle aspiration (EUS-FNA) of solid pancreatic masses. Am J Gastroenterol 2011; 106: 1705-1710

[44] Hocke M, Topalidis T, Braden B et al. "Clinical” cytology for endoscopists: A practical guide. Endosc Ultrasound 2017; 6: 83-89

[45] Biermann K, Escario MDL, Hebert-Magee $S$ et al. How to prepare, handle, read, and improve EUS-FNA and fine-needle biopsy for solid pancreatic lesions: The pathologist's role. Endoscopic Ultrasound 2017; 6: S95-S98

[46] Chen J, Yang R, Lu Y et al. Diagnostic accuracy of endoscopic ultrasound-guided fine-needle aspiration for solid pancreatic lesion: a systematic review. J Cancer Res Clin Oncol 2012; 138: 1433-1441

[47] Hébert-Magee S, Bae S, Varadarajulu S et al. The presence of a cytopathologist increases the diagnostic accuracy of endoscopic ultrasound-guided fine needle aspiration cytology for pancreatic adenocarcinoma: a meta-analysis. Cytopathology 2013; 24: 159-171

[48] van Riet PA, Cahen DL, Poley JW et al. Mapping international practice patterns in EUS-guided tissue sampling: outcome of a global survey. Endosc Int Open 2016; 4: E360-E370

[49] Biscotti CV, Hollow JA, Toddy SM et al. ThinPrep versus conventional smear cytologic preparations in the analysis of thyroid fine-needle aspiration specimens. Am J Clinic Pathol 1995; 104: 150-153

[50] Rossi ED, Raffaelli M, Zannoni GF et al. Diagnostic efficacy of conventional as compared to liquid-based cytology in thyroid lesions: evaluation of 10,360 fine needle aspiration cytology cases. Acta Cytolog 2009; 53: 659-666

[51] Michael CW. Liquid-Based Cytology Technique for Thyroid Cytology . In Thyroid FNA Cytology: Springer2019: 101-112

[52] Rivera R, Madera J. EP1086 Diagnostic accuracy of conventional cervical cytology (Papanicolau smear), liquid based cytology and visual inspection with acetic acid in detecting premalignant and malignant cervical lesions among Filipino women in a tertiary hospital. BM] Special J 2019; 29: A569

[53] Hing E, Saraiya M, Roland K. Liquid-based cytology test use by officebased physicians: United States, 2006-2007. Nat Health Stat Rep 2011; 40: 1-6

[54] Arbyn M, Herbert A, Schenck U et al. European guidelines for quality assurance in cervical cancer screening: recommendations for collecting samples for conventional and liquid-based cytology. Cytopathology 2007; 18: 133-139

[55] Hoda RS, VandenBussche C, Hoda SA. Liquid-Based Specimen Collection, Preparation, and Morphology. In, Diagnostic Liquid-Based Cytology: Springer2017: 1-12

[56] Nayar R, Wilbur DC. The Bethesda System for Reporting Cervical Cytology. Acta Cytol 2017; 61: 359-372

[57] Mitoro A, Nishikawa T, Yoshida M et al. Diagnostic efficacy of liquidbased cytology in endoscopic ultrasound-guided fine needle aspiration for pancreatic mass lesions during the learning curve: a retrospective study. Pancreas 2019; 48: 686-689

[58] Gao L, Zhang M, He X et al. Liquid-based cytology diagnosis of endoscopic ultrasound-guided fine needle aspiration of pancreatic lesions. Chinese J Pathol 2016; 45: 43-46

[59] Ohtani M, Ofuji K, Nosaka T et al. Diagnostic utility of liquid-based cytology in EUS-FNA for pancreatic lesion and subepithelial tumor. Gastrointest Endosc 2018; 87: AB340

[60] Pannala R, Hallberg-Wallace KM, Smith AL et al. Endoscopic ultrasound-guided fine needle aspiration cytology of metastatic renal cell carcinoma to the pancreas: A multi-center experience. Cytojournal 2016; 13: 24 